\title{
Exercício físico no tratamento da hipertensão: a propósito de um caso
}

António Cruz-Ferreira, Estela Loureiro, ${ }^{* *}$ Iva Pimentel***

\section{RESUMO}

O sedentarismo representa um importante problema na sociedade e é sabido que se associa a um risco acrescido de hipertensão arterial (HTA) e outras comorbilidades cardiovasculares. Na abordagem da maioria das doenças associadas a factores de risco ambientais, a prescrição do exercício físico regular constitui um recurso fundamental que deve ser prescrito pelos médicos de família, como primeira linha terapêutica.

Apresenta-se o caso clínico de uma mulher de 60 anos, sedentária, que recorreu à médica de família por elevação nos valores da tensão arterial (TA) medidos no domicílio. Foi-lhe prescrito um programa individual que visava a alteração do estilo de vida e a prática regular de exercício físico aeróbio de forma estruturada. Após 6 meses de tratamento, a doente apresentou-se com valores tensionais normalizados não tendo sido necessário, até ao momento, a introdução de qualquer medicação anti-hipertensora.

Através do relato deste caso de sucesso, procura-se incentivar/encorajar os médicos de família à prescrição da prática regular de exercício físico aeróbio no tratamento dos seus doentes hipertensos.

Palavras-chave: Exercício Físico; Hipertensão Arterial; Sedentarismo.

\section{INTRODUÇÃO}

1 HTA é um problema de saúde pública dos países industrializados atingindo, actualmente, mais de $25 \%$ da população. ${ }^{1-4}$ Quando não tratada, a HTA pode causar lesões em múltiplos órgãos, associando-se a um aumento da mortalidade e da morbilidade..$^{1-3}$

Sabe-se, também, que o sedentarismo é um grave problema das sociedades modernas e que está relacionado com um aumento de risco de HTA, obesidade, diabetes mellitus e suas comorbilidades. ${ }^{1-5} \mathrm{Na}$ verdade, estima-se que menos de $50 \%$ dos cidadãos, na União Europeia, estão envolvidos em actividades físicas aeróbias regulares, de lazer ou profissionais. ${ }^{6}$

A prática regular de exercício físico de intensidade moderada está associada a uma redução da tensão arterial sistólica e diastólica muito semelhante à da mono-

* Interno de Medicina Geral e Familiar, UCSP da Mealhada

**Interna de Medicina Geral e Familiar, UCSP da Mealhada

***Assistente de Medicina Geral e Familiar, UCSP da Mealhada terapia anti-hipertensora farmacológica $(10 \mathrm{mmHg}$ e 5 $\mathrm{mmHg}$, respectivamente), ${ }^{1,7-11}$ bem como a redução de peso, perímetro abdominal e a resistência à insulina. ${ }^{7-10,12}$

Por outro lado, sabemos que a instituição de programas de alteração dos estilos de vida que incluam a restrição do consumo de sal (para menos de seis gramas por dia), a adopção de uma dieta variada, nutricionalmente equilibrada, rica em legumes, leguminosas, verduras e frutas e pobre em gorduras, bem como a prática regular e continuada de exercício físico e a moderação do consumo de álcool são, para a Sociedade Europeia de Cardiologia (SEC), recomendações de classe I e nível de evidência B, de acordo com as «Guidelines Europeias para a Prevenção de Doenças Cardiovasculares na Prática Clínica 2012». ${ }^{7}$ Esta mesma sociedade europeia preconiza a necessidade de iniciar tratamento farmacológico apenas para os casos de HTA de grau II e III, ou a partir de grau I quando associado a risco cardiovascular elevado ou muito elevado (Quadros I e II). ${ }^{7,11}$ Nos casos de HTA de grau I com baixo risco cardiovascular pode-se optar por protelar a introdução de terapêutica farmacoló- 
gica por alguns meses, e no grau II com baixo ou moderado risco cardiovascular por algumas semanas, desde que se instituam alterações do estilo de vida e a vigilância dos valores tensionais. ${ }^{7,8}$

As grandes sociedades internacionais de cardiologia acreditam que a implementação e sucesso dos programas de prevenção de doenças cardiovasculares na Europa, bem como a adesão aos planos terapêuticos propostos dependem, fundamentalmente, dos médicos de família. ${ }^{7,8,13} \mathrm{Na}$ maioria dos países, como em Portugal, $90 \%$ das consultas são feitas no âmbito dos Cuidados de Saúde Primário (CSP), incluindo cuidados preventivos, rastreio e gestão de doenças crónicas e acompanhamento geral. ${ }^{7}$ No caso da prevenção de Doenças Cardiovasculares (DCV), os médicos de família têmum papelúnico na identificação de indivíduos em risco, diagnóstico e intervenção precoce. ${ }^{7}$ Por outro lado, as próprias características dos CSP (maior proximidade física e mais fácil estabelecimento de uma relação médico-doente duradora e de confiança), aliadas a estratégias de promoção da adesão à terapêutica, como motivação, responsabilização, manutenção em consultas de seguimento ou contactos informais, poderão resultar num melhor cumprimento terapêutico eno sucesso dasintervenções, ultrapassando as crónicas barreiras à modificação de estilos de vida. $^{8,13}$

Segue-se a descrição do caso clínico de uma mulher com marcados hábitos sedentários e valores de TA elevados a quem foi proposto um programa de alteração de estilos de vida, no qual estava incluída a prática regular de exercício físico. Dada a importância de promover a prática regular de exercício físico, no âmbito de programas mais alargados de modificação de estilos de vida, bem como a relevância que a HTA e as DCV têm na sociedade portuguesa, pareceu-nos pertinente descrever este caso, alertando para a importância de prescrever, de forma sistematizada, o exercício físico como arma terapêutica no doente hipertenso.

\section{DESCRIÇÃO DO CASO}

Relatamos o caso de uma mulher de 60 anos, casada, com dois filhos adultos, licenciada em Enfermagem e recentemente reformada, que recorreu à consulta de saúde de adultos da sua médica de família, em Dezembro de 2011. Pertence a uma família nuclear, de classe média-alta, na fase VI do ciclo de vida de Duvall e com um APGAR familiar de 10.

Primeira consulta (Dezembro 2011): veio por apresentar, em medições no domicílio, tensões arteriais sistólicas (TAS) superiores a $150 \mathrm{mmHg}$ e diastólicas (TAD) a $90 \mathrm{mmHg}$. A iniciativa de monitorizar a sua TA deveu-se a um achado ocasional, 2 meses antes, quando em casa mediu a sua TA após ter avaliado a do seu pai, tendo registado um valor de $155 \mathrm{mmHg}$ de TAS e $90 \mathrm{mmHg}$

\begin{tabular}{|c|c|c|}
\hline Classificação & Tensão arterial sistólica & Tensão arterial diastólica \\
\hline Normal & $120-129 \mathrm{mmHg}$ & $80-84 \mathrm{mmHg}$ \\
\hline Normal Alta & $130-139 \mathrm{mmHg}$ & $85-89 \mathrm{mmHg}$ \\
\hline HTA Grau I - ligeira & $140-159 \mathrm{mmHg}$ & $90-99 \mathrm{mmHg}$ \\
\hline HTA Grau II - moderada & $160-179 \mathrm{mmHg}$ & $100-109 \mathrm{mmHg}$ \\
\hline HTA Grau III - grave & $\geq 180 \mathrm{mmHg}$ & $\geq 110 \mathrm{mmHg}$ \\
\hline
\end{tabular}

QUADRO II. Estratificação do risco cardiovascular (RCV) de acordo com as linhas de orientação da SEC (adaptado de European Guidelines on cardiovascular disease prevention in clinical practice - 2012). ${ }^{7}$

\begin{tabular}{l|l} 
Classificação & \multicolumn{1}{c}{ Critérios } \\
\hline Risco muito elevado & $\begin{array}{l}\text { Uma das seguintes características: } \\
\text { DCV documentada } \\
\text { Diabetes mellitus }+\geq 1 \text { factor de RCV e/ou dano em órgão-alvo } \\
\text { Doença renal crónica grave } \\
\text { Resultado SCORE } \geq 10 \% .\end{array}$ \\
\hline Risco elevado & $\begin{array}{l}\text { Uma das seguintes características: } \\
\text { Dislipidemia familiar e/ou hipertensão grave. } \\
\text { Diabetes mellitus, sem fatores de RCV ou lesão de órgão-alvo } \\
\text { Doença renal crónica moderada } \\
\text { Resultado SCORE } \geq 5 \%-<10 \%\end{array}$ \\
\hline Risco moderado & Resultado SCORE $\geq 1-<5 \%$ \\
\hline Risco baixo & Resultado SCORE $<1 \%$ \\
\hline
\end{tabular}


de TAD. Assintomática, tinha antecedentes pessoais de sedentarismo e excesso de peso. Em relação aos seus antecedentes familiares, há a referir a HTA da mãe e a diabetes mellitus tipo 2 de uma irmã. A doente referiu, do ponto de vista alimentar, que fazia uma dieta equilibrada, sem consumo excessivo de fritos ou hidratos de carbono de absorção rápida, negava consumir sal de forma exagerada (apesar de não conseguir objectivar a quantidade exacta), álcool ou tabaco. Quanto aos hábitos de exercício físico, negava qualquer prática regular de actividade física estruturada e planeada nos últimos 10 anos.

Ao exame objectivo, para além de uma TA elevada, registámos uma estatura de 1,60 m, $75 \mathrm{~kg}$ de peso e um índice de massa corporal (IMC) de $29,3 \mathrm{~kg} / \mathrm{m} 2$, bem como um perímetro abdominal de $81 \mathrm{~cm}$. A tensão arterial foi medida em ambos os braços e no membro inferior direito, recorrendo a um esfigmomanómetro manual e a um electrónico. As medições efectuadas revelaram uma TAS média de 150 mmHg e TAD média de 95 mmHg. Repetiu-se a medição da TA, passados 15 minutos, tendo-se confirmado os valores anteriores, agora com 150 mmHg de TAS e 90 mmHg de TAD.

A doente era, igualmente, portadora de controlo analítico recente (Outubro de 2011) sem alterações relevantes. Com os dados obtidos pôde-se calcular o risco cardiovascular a 10 anos da doente, com recurso ao modelo de avaliação de risco SCORE (1,6\% de risco global, 0,7\% de doença coronária e $0,9 \%$ de doença não coronária). ${ }^{14}$ Concluímos, assim, estar na presença de uma doente sedentária, com excesso de peso, obesidade abdominal, HTA de grau I, um risco cardiovascular moderado (entre 1 e 5\%) e uma função renal adequada para a idade.

Em função dos dados obtidos, seguindo as orientações da Direcção-Geral de Saúde (DGS) e indo de encontro às da SEC,,$^{3,7}$ foi explorada a possibilidade de se optar por um conjunto de alterações no estilo de vida e a reavaliação da TA dentro de 2 meses. Em função da aparente adesão à ideia e da manifestação de que esta opção era preferível a ter de tomar medicação, foram reforçadas as recomendações de restrição do consumo de sal (para menos de seis gramas por dia), a adopção de uma dieta variada, nutricionalmente equilibrada, rica em legumes, leguminosas, verduras e frutas e pobre em gorduras, bem como a prática regular e continuada de exercício físico. ${ }^{3,7}$ Em relação à prescrição do exercício físico, seguindo as orientações recentes do American College of Sports Medicine (ACSM) recomendou-se a realização de pelo menos cinco períodos semanais de 30 a 60 minutos de exercício físico aeróbio de intensidade moderada (caminhada na passadeira, por opção da doente). A intensidade do exercício foi calculada em função de uma percentagem da sua frequência cardíaca máxima (FCmáx), calculada pela fórmula: [220-idade*0.7]. ${ }^{15}$ Neste caso, prescreveu-se a realização de exercício físico a $70 \%$ da sua FCmáx, que corresponde a 116 pulsações por minuto. Por forma a garantir uma maior adesão à terapêutica proposta, a equipa médica elaborou um esquema/resumo de tudo o que tinha sido proposto e entregou-o à doente deixando, posteriormente, espaço para a colocação de dúvidas que foram prontamente esclarecidas. Foi, igualmente, marcada nova consulta para Março de 2012, tendo sido negociado com a doente o cumprimento de algumas metas: redução do peso em pelo menos $1 \mathrm{~kg}$, realização de pelo menos três períodos semanais de exercício físico nos moldes propostos e a manutenção dos registos semanais da TA. Para complementar a avaliação inicial desta doente, seguindo as orientações da DGS, foi pedido um electrocardiograma (ECG) e o doseamento da microalbuminúria.

Segunda consulta (Março de 2012): a doente regressou à nossa consulta para reavaliação da sua TA e das metas de redução de peso e alteração de estilos de vida instituídos. Quanto às recomendações dietéticas, tendo em conta que as mesmas foram, de acordo com a doente, ao encontro da sua alimentação normal, não foram registadas alterações. A doente referiu manter uma alimentação variada, equilibrada e com baixo consumo de sal, tal como já referia antes da instituição do programa terapêutico. Afirmou, todavia, que tinha conseguido cumprir um mínimo de três períodos semanais de exercício físico, de acordo com o estabelecido na consulta anterior. Referia, também, que tinha começado a frequentar um ginásio da sua área de residência, onde estabeleceu uma relação de proximidade com os monitores e alguns utentes que também se encontravam reformados e a praticar exercício físico como parte de um programa terapêutico individual dirigido aos seus problemas de saúde. Informou-nos, igualmente, da redução média nos valores de TA medidos na domicílio, que agora eram de $135 \mathrm{mmHg}$ de TAS e a $85 \mathrm{mmHg}$ de TAD. Ao exame objectivo, para além da diminuição de $1,5 \mathrm{~kg}$ de peso corporal (agora $73,5 \mathrm{~kg}$, que se traduzia num IMC de $28,7 \mathrm{~kg} / \mathrm{m}^{2}$ ) e do perímetro abdominal (agora 79,5 cm), verificou-se a redução dos valores da TAS média no consultório para $135 \mathrm{mmHg}$ e $85 \mathrm{mmHg}$ de TAD. O ECG e a microalbuminúria pedidas não apresentavam alterações. A doente foi inquirida sobre eventuais dificuldades no cumprimento do programa terapêutico instituído, tendo refe- 
rido que apenas no plano das alterações dos hábitos alimentares não tinha feito qualquer modificação. Todavia, tendo em conta a positiva evolução do peso corporal, optou-se por não impor qualquer modificação alimentar, destacando o entusiasmo pelos resultados obtidos, a necessidade de manutenção do programa terapêutico e as suas vantagens para a saúde da doente, tendo sido marcada nova consulta para Maio de 2012.

Terceira consulta (Maio de 2012): houve novo contacto para reavaliação do programa terapêutico e das metas alcançadas. Desta vez, apesar de não terem sido negociados objectivos terapêuticos, a doente referiu manter o mesmo plano de exercício físico e, ao exame objectivo, apresentava valores médios de TAS de $138 \mathrm{mmHg}$ e de TAD de 84 $\mathrm{mmHg}$, consonantes com os obtidos na segunda consulta. Em relação ao peso corporal e ao perímetro abdominal não foram detectadas alterações significativas $(73 \mathrm{~kg}$ de peso e $79 \mathrm{~cm}$ de perímetro abdominal). Foram, novamente, exploradas eventuais dificuldades de adesão à terapêutica ou barreiras/obstáculos à mesma. A doente negou qualquer dificuldade, admitindo, inclusivamente, que tinha ganho novos hábitos, tendo passado a frequentar, todas as manhãs, o seu ginásio. Esta rotina permitia-lhe, para além de cumprir o esquema terapêutico proposto, socializar com outras pessoas, algumas também a cumprir esquemas terapêuticos de exercício físico e, desta forma, pela «coesão intergrupal» e partilha de objetivos, auto-motivarem-se para alcançar as metas estabelecidas. Neste momento, a doente continua a realizar o programa terapêutico instituído e a frequentar as consultas nos CSP com periodicidade semestral.

\section{COMENTÁRIO}

Como já foi referido, a HTA é um problema dos países desenvolvidos, contribuindo para um aumento da morbilidade e mortalidade nessas populações. ${ }^{1-4}$ Sem qualquer margem de dúvida, o sedentarismo, fruto do ritmo de vida das populações, é um problema das sociedade modernas. Este associa-se a um conjunto de problemas de saúde e deverá ser activamente combatido pelos profissionais da área, começando pelos médicos de família. ${ }^{1-4}$ Como já referido, cabe ao médico de família um papel único na identificação de indivíduos em risco, diagnóstico e intervenção precoce. ${ }^{7}$ A própria SEC reconhece que a maior proximidade física e a relação médico-doente única dos CSP poderão resultar num melhor cumprimento terapêutico e sucesso das intervenções. Para tal, torna-se necessário implementar um conjunto de estratégias de promoção da adesão à terapêutica, como a motivação, responsabilização, manutenção em consultas de seguimento ou contactos informais, ultrapassando conhecidas barreiras à modificação de estilos de vida. ${ }^{8,13}$

As recentes normas da DGS e da SEC para o tratamento da HTA apontam a modificação do estilo de vida como primeira linha no tratamento da HTA, sendo uma das mudanças propostas o início da prática regular de exercício físico ( 30 a 60 minutos por dia, quatro a sete dias por semana). ${ }^{3}$ Os efeitos na diminuição da resistência vascular periférica e no aumento na sensibilidade barorreflexa, relacionados com uma diminuição na actividade do sistema nervoso simpático, com a bradicardia em repouso, diminuição do volume plasmático e do volume sistólico, que podem levar à diminuição do débito cardíaco, são os motivos que conduzem à prescrição do exercício físico aeróbio regular como primeira linha de tratamento da HTA. ${ }^{1,2,4}$ Para facilitar a prescrição de exercício físico nestes doentes e, de acordo com a ACSM, o médico assistente deve optar por o fazer segundo a fórmula FITT [frequência(F), intensidade(I), Tempo(T) e Tipo(T)], nos moldes sugeridos pelo Quadro III. ${ }^{2}$

Os efeitos benéficos da prática regular de exercício físico já foram aqui descritos. No entanto, sempre que se prescreve o exercício físico como parte de um conjunto de modificações do estilo de vida, não se podem nunca dissociar os resultados obtidos dos da modificação na dieta, na ingestão de sal e na moderação do hábitos etílicos e tabágicos que possam ter sido, igualmente, implementados. No caso que descrevemos, a diminuição dos valores de TA registados, embora ligeira, parece resultar do programa terapêutico instituído, no qual o exercício físico assume particular importância. A pronta adesão desta doente ao programa de exercício pres-

$\begin{aligned} & \text { QUADRO III. Prescrição de exercício físico ao doente hipertenso (adaptado de ACSM Guidelines } \\
& \text { for exercise testing and prescription). }\end{aligned}$
\begin{tabular}{l|c|c|c} 
Frequência (F) & Intensidade (I) & Tempo (T) & Tipo (T) \\
\hline 5-7 dias/semana & 50 a $75 \%$ da FCmáx & 30 a 60 minutos & $\begin{array}{l}\text { Caminhada, passadeira, ciclismo, bicicleta } \\
\text { estática, remo indoor, natação. }\end{array}$ \\
\hline
\end{tabular}


crito, o que nem sempre se verifica no âmbito dos CSP, poderá ter-se devido às relações interpessoais estabelecidas no ginásio que passou a frequentar mas, também, pelo facto de se tratar de uma ex-profissional de saúde e, desta forma, estar mais atenta para a necessidade de cumprimento do programa e dos riscos associados à HTA. A identificação de um caso de sucesso, apesar de merecer destaque e ser motivo de divulgação, funcionando como estímulo a outros profissionais de saúde, não pode deixar de ser analisado de forma individual e sem que daí se possa generalizar. Desta forma, serve o presente caso para demonstrar que, apesar das habituais dificuldades de adesão a programas de mudança de estilos de vida, existem alguns casos em que será possível fazer esta opção, desde que todos os riscos tenham sido devidamente avaliados e cumprindo, sempre, as linhas de orientação em vigor.

\section{REFERÊNCIAS BIBLIOGRÁFICAS}

1. Mancia G, De Backer G, Dominiczak A, Cifkova R, Fagard R, Germano G, et al. 2007 European Guidelines for the management of arterial hypertension. Eur Heart J 2007 Jun; 28 (12): 1462-536.

2. American College of Sports Medicine (ACSM). ACSM's Guidelines for exercise testing and prescription. 8th ed. Philadelphia: LippincottWilliams \&Wilkins; 2010. p.249-50.

3. Direcção Geral de Saúde (DGS). Abordagem Terapêutica da Hipertensão Arterial. Norma nº 026/2011. Lisboa: DGS; 2011.

4. Rondon MU, Brum PC. Exercício físico como tratamento não-farmacológico da hipertensão arterial. Rev Bras Hipertens 2003 Abr-Jun; 10 (2): 134-9.

5. Fry J, Finley W. The prevalence and costs of obesity in the EU. Proc Nutr Soc 2005 Aug; 64 (3): 359-62.

6. Afonso C, Graça P, Kearney JM, Gibney MJ, de Almeida MD. Physical activity in European seniors: attitudes, beliefs and levels. J Nutr Health Aging 2001; 5 (4): 226-9.
7. Perk J, De Backer G, Gohlke H, Graham I, Reiner Z, Verschuren M, et al. European Guidelines on cardiovascular disease prevention in clinical practice (version 2012). Eur Heart J 2012 Sep, 33 (17): 1635-1701.

8. Canadian Hypertension Education Program, Campbell N. 2011 Canadian Hypertension Education Program recommendations: an annual update. Can Fam Physician 2011 Dec; 57 (12): 1393-7.

9. Gómez R, Monteiro H, Cossio-Bolaños MA, Fama-Cortez D, Zanesco A. Perscripción del ejercicio físico. Rev Peru Med Exp Salud Publica 2010 Sep; 27 (3): 379-86.

10. Veríssimo MT. HTA e exercício físico. Rev Med Desp informa 2011; 2 (2): 10-2.

11. Dimeo F, Pagonas N, Seibert F, Arndt R, Zidek W, Westhoff TH. Aerobic exercise reduces blood pressure in resistant hypertension. Hypertension 2012 Sep; 60 (3): 653-8.

12. Rossi A, Dikareva A, Bacon SL, Daskalopoulou SS. The impact of physical activity on mortality in patients with high blood pressure: a systematic review. J Hypertens 2012 Jul; 30 (7): 1277-88.

13. Jones DE, Carson KA, Bleich SN, Cooper LA. Patient trust in physicians and adoption of lifestyle behaviors to control high blood pressure. Patient Educ Couns 2012 Oct; 89 (1): 57-62.

14. Conroy RM, Pyörälä K, Fitzgerald AP, Sans S, Menotti A, De Backer G, et al. Estimation of ten-year risk of fatal cardiovascular disease in Europe: the SCORE project. Eur Heart J 2003 Jun; 24 (11): 987-1003.

15. Tanaka H, Monahan KD, Seals DR. Age-predicted maximal heart rate revisited. J Am Coll Cardiol 2001 Jan; 37 (1): 153-6.

\section{CONFLITOS DE INTERESSE}

Os autores declaram não possuir qualquer tipo de conflitos de interesse.

\author{
ENDEREÇO PARA CORRESPONDÊNCIA \\ António Miguel da Cruz Ferreira \\ Rua Principal - Paredes S/N \\ 3020-285 Coimbra \\ E-mail: krusferreira@hotmail.com
}

Recebido em 11/06/2012

Aceite para publicação em 08/03/2013

\section{ABSTRACT}

\section{EXERCISE IN THE MANAGEMENT OF HYPERTENSION: A CASE REPORT}

Physical inactivity is a problem in modern society and its association with hypertension and other cardiovascular diseases is well known. In treating diseases associated with environmental risk factors, the prescription of regular exercise is a tool that can be used by family physicians.

We report the case of a 60-year-old sedentary woman who came to her family doctor after measuring elevated blood pressure at home. An individual treatment program was prescribed, consisting of lifestyle changes and promotion of regular aerobic exercise. After 6 months of treatment, the patient had normal blood pressure values and no anti-hypertensive drugs were required.

We want to encourage family physicians to prescribe regular aerobic exercise as a first line or adjuvant treatment in hypertensive patients.

Keywords: Exercise; Hypertension; Sedentarism. 